\title{
IL-2, IL-15 and IL-21 expand T cells for targeted adoptive therapy
}

\author{
Zhenjiang Liu, Qingda Meng, Markus Maeurer ${ }^{*}$, Elena Rangelova, Thomas Poiret, Rebecca Robertson, \\ Aditya Ambati, Lalit Rane, Jiri Bartek, Caroline Verbeke, Oscar Persson, Matthias Löhr, Ralf Segersvärd, \\ Ernest Dodoo
}

From 30th Annual Meeting and Associated Programs of the Society for Immunotherapy of Cancer (SITC 2015) National Harbor, MD, USA. 4-8 November 2015

\section{Background}

Expansion of antigen-specific $\mathrm{T}$ cells, from peripheral blood specific for tumor-associated antigens (TAAs) is a prerequisite for the advanced cellular therapy. Such antigen-specific $\mathrm{T}$ cells should express a Th1-functional phenotype and are able to enter tumor-tissue. We identified a cytokine cocktail, comprised of IL-2, IL-15 and IL-21 that drives antigen-specific T cells from peripheral blood mononuclear cells to tumor-associated antigens.

\section{Materials and methods}

20 blood samples ( 3 from health donors, 11 from glioma patients and 6 from pancreatic cancer patients) were collected, $\mathrm{T}$ cells were expanded using the cytokine cocktail IL-2/IL-15/IL-21 and TAAs, e.g. NY-ESO-1 or infectious antigens, e.g. CMV pp65 within 18-21 days. Intracellular Cytokine Staining (ICS) was used to detect antigen-specific immune responses by combining CD3, CD4 and CD8 markers with IL-2, TNF- $\alpha$, IFN- $\gamma$ production. Chemokine markers like CCR4, CCR6 or CXCR3 were used for phenotyping distinguishing Th1 and Th2 subtypes in CD4 + T cells, as well as in CD3+, CD4-CD8- (double-negative) $\mathrm{T}$ cell subsets. $\mathrm{T}$ cell memory phenotypes were defined by CCR7 and CD45RA staining. Tetramer (NY-ESO-1 or CMV specific) staining were used to show expansion of antigen specific $\mathrm{T}$ cells using a panel of mutant MHC class I tetramer molecules that allow to gauge for high, intermediate and low-affinity $\mathrm{T}$ cell populations imposed by interference of the MHC class I heavy chain with the $\mathrm{CD} 8 \alpha$ binding site.

\section{Results}

$\mathrm{T}$ cells from peripheral blood could be expanded (up to $10 \mathrm{e} 10$ cells) using IL-2, IL-15 and IL-21 and TAAspecific CD8, CD4 and CD3+, CD4-CD8-T cells could reliably be expanded defined by intracellular cytokine staining. In general, Th1 cells (CCR4-CXCR3+CCR6-) could be readily expanded along with (i.e. from $22.2 \%$ to 92.1\%) along with increased expression of CXCR3 in both CD8+ and CD4+ T cells that enables increased access to tumor tissue. MHC class I-reactive T cells, directed against single $\mathrm{T}$ cell epitopes could be observed in up to $10 \%$ of CD8+, NY-ESO-1 specific T cells with a strong expansion of 'high affinity' $\mathrm{T}$ cells defined by i) mutant tetramers that interfere with CD8 engagement and ii) TAA-reactive $\mathrm{T}$ cells in the CD3+, CD4-CD8double negative $\mathrm{T}$ cell subset.

\section{Conclusions}

$\mathrm{T}$ cells from peripheral blood samples can be reliably and successfully expanded in IL-2, IL-15 and IL-21, they show high affinity TCR - MHC class I/peptide engagement, a Th1-cytokine production pattern and increased CXCR3 expressing allowing $\mathrm{T}$ cell access into tissues. A Phase I clinical trial to target TAAs in patients with glioblastoma or pancreatic cancer will soon start at Karolinska using IL-2/IL-15/IL-21 and TAA-expanded T cells.

Published: 4 November 2015

doi:10.1186/2051-1426-3-S2-P31

Cite this article as: Liu et al:: IL-2, IL-15 and IL-21 expand T cells for targeted adoptive therapy. Journal for ImmunoTherapy of Cancer 20153 (Suppl 2):P31.

Karolinska Institutet, Stockholm, Sweden original work is properly cited. The Creative Commons Public Domain Dedication waiver (http://creativecommons.org/publicdomain/ zero/1.0//) applies to the data made available in this article, unless otherwise stated. 